\title{
International Ultraviolet Explorer to be launched this week
}

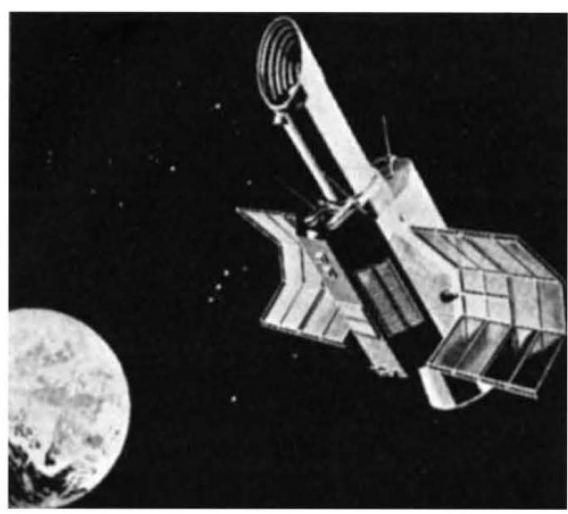

DURING the course of evolution some animals have developed eyes on stalks as a rather bizarre adaptation to their environment. Astronomers could well do the same to escape the disturbing effects of the earth's atmosphere but evolution is a slow process and, luckily, there are mone acceptable solutions. For many years now, astronomers working at wavelengths that are absorbed by the atmosphene have been sending probes into space, but the available techniques have not been entirely satisfactory. Rockets and balloons provide only fleeting glimpses and orbiting satellites not continually in communication with the ground must be automated to collect data blindly for later transmission. A solution is requined which enables the astronomer continuously to control and monitor the performance of a telescope that nemains above the atmosphere for long periods of time.

That solution is provided for the ultraviolet (UV) waveliength region by the International Ultnaviolet Explorer (IUE) satellite scheduled for launch into geosynchronous orbit on 26 January. The satellite will be continuously visible from a control station on the ground and can be used just as if it were a ground-based UV telescope not suffering from the effects of the atmosphere.

In the ultraviolet region those effects are disastrous. The earth's atmosphere becomes incoeasingly opaque at wavelengths below 4,000 $\AA$ and is effectively 'black' beyond 3,000 $\AA$. Balloons can abserve over most of the mid-ultraviolet range from $3,000 \AA$ to $2,000 \AA$ by ascending above the bulk of the ozone layer to heights of $40 \mathrm{~km}$, but for the far ultraviolet below $2,000 \AA$ it is necessary to go much higher. The planned orbit of IUE varies from $25,000 \mathrm{~km}$ to $46,000 \mathrm{~km}$ altitude.

There are sound reasons for wanting information at ultraviolet wavelengths. The sun is brighter at visible wavelengths than in the far ultraviolet but this is not true for many other stars. As the temperature of a source increases, the emitted radiation appears at shorter wavelengths and at $20,000 \mathrm{~K}$, a typical surface temperature of a newly-formed star, almost all of the light is at wavelengths that cannot penetrate the earth's atmosphere.

The cooler negions between the stars are also impontant at UV wavelengths. Interstellar gas contains about $10 \%$ of the material in our galaxy and can be detected only by the characteristic absorption lines it imposes on the spectra of objects observed through it. The gas constituents are in their lowest energy state because of the low densities and temperatures in space and so it is necessary to observe at wavelengths where the atoms and molecules in their ground states can absorb light.

The common elements in interstellar gas are hydrogen, helium, oxygen, carbon, nitrogen and silicon. They all have their strongest absorption lines below $3,000 \AA$, with only the less abundant sodium and calcium appearing in the visible region. Therefore the major constituents of interstellar gas clouds can only be observed by measurements in the UV. If the gas clouds are moving, then the absorption lines will be Doppler shifted in wavelength and high resolution measurements of the spectra allow their velocity to be calculated.

Curiously, UV emission from some of the stranger objects in the sky has been studied from the ground. Many of the immensely distant quasars are receding so fast that their UV light is Doppler shifted into the visible region; they are saiid to have large redshifts. These cannot be compared directly with low redshift quasars whose UV emission is shifted less and still remains in the band absorbed by the atmosphere. Direct comparision is essential to find out if quasars are at cosmological distances, and the Lyman alpha line is crucial as it may provide a measure of the intrinsic brightness of quasars. So far, quasars have been disappointing cosmological objects simply because they could not be easily observed in the ultraviolet. But a recent UV observation of a low medshift quasar has imposed impontant constraints on cosmological models. Those measurements were made from a rocket flight and lasted only four minutes-IUE can observe for 24 hours a day.

The present knowledge of the appearance of the universe in the UV comes from balloon and rocket flights and from experiments on board the American Orbiting Astronomical Observatory saitellites OAO-2 and OAO-3 (renamed Copernicus) the Dutch ANS satellite and the European TD-1 satellite. IUE is dedicated solely to UV observations and promises an enormous improvement on the earlier, largely survey, experiments. The IUE project is itself a forerunner for the Space Telescope planned to be launched from the Space Shuttle in 1983.

There are two broad aims of IUE and these impose conflicting nequirements on the instrumentation. The measurement of chemical abundances, and the study of gas motions, requine a detailed study of line profiles with very high spectral resolution. But IUE should be able to deteat faint sources making necessary good light collection efficiency rather than resolving power. The ability to observe for llong periods also means that variable phenomena can be observed.

To satisfy these nequirement, IUE carries a $1.3 \mathrm{~m}$ long, $45-\mathrm{cm}$ diameter aperture, Cassegrain telescope which will be used exclusively for spectroscopy. No direct imaging will be made in the UV although a visible-light picture will be used for target identification. Light from the telescope is passed through two spectrographs, each containing a high dispersion echelle grating in series with a low dispersion grating; two spectrographs are needed to achieve resolutions of between $0.1 \AA$ and $0.2 \AA$ over the full wavelength range of $1,150 \AA$ to $3,200 \AA$. For the low resolution mode, the echelle is simply bypassed, producing a spectrograph that can be used to record stars as faint as 14 th magnitude with a $6 \AA$ spectral resolution.

IUE is a joint venture between the US National Aeronautics and Space Administration (NASA), the European Space Agency (ESA), and the UK Science Research Council (SRC). The major contributor is NASA. ESA is providing the solar panels and one of the two ground stations and the SRC is supplying the UV sensitive television cameras used for spectra detectors. The observing time on IUE will be shared on a daily basis with 16 hours going to NASA, and 4 hours each to ESA and the SRC who have agreed to take 8 hour periods on alternate days.

As the telescope is in geosynchronous orbit it will be in continuous contact with the NASA ground station at the Goddard Space Flight Center and will be in view at least 10 hours a day from the ESA ground station at Villafranca near Madrid. This arrangement allows real-time operation of the telescope and reduces the training time necessary for observers. As a result, the telescope can be opened up for international use and guest observens are encouraged to use IUE in a similar fashion to ground based telescopes.

Stuart Sharrock 